However, centres prescribing complete, intermediate and incomplete work-ups were differently represented for the two diagnoses. No statistically significant change emerged in diagnosis, diagnostic confidence or clinical management between complete, intermediate or incomplete assessments (Table). Stratifying patients for etiopathology (AD-FTD) or clinical severity (MCI-dementia) led to the same results. Conclusions: Collection of additional core bio-markers does not seem to affect the incremental value of amyloid-PET in naturalistic clinical setting. The clinicians combinations and use of instrumental examination needs to be better understood and elucidated in view of the definition of an evidence-based diagnostic algorithm.

\section{O2-08-05 NEURONAL INJURY AND DEGENERATION EVALUATED WITH IMAGING AND CSF BIOMARKERS IN AUTOSOMAL DOMINANT ALZHEIMER'S DISEASE: RESULTS FROM THE DIAN STUDY}

Nelly Joseph-Mathurin ${ }^{1}$, Andrei G. Vlassenko ${ }^{1}$, Anne M. Fagan ${ }^{1}$, Yi Su ${ }^{1}$, Karl A. Friedrichsen ${ }^{1}$, Christopher J. Owen ${ }^{1}$, Brian A. Gordon ${ }^{1}$,

Russ C. Hornbeck ${ }^{1}$, Robert A. Koeppe ${ }^{2}$, Chengjie Xiong ${ }^{1}$, John C. Morris ${ }^{1}$, Randall Bateman ${ }^{1}$, Tammie LS. Benzinger ${ }^{1}$, Dominantly Inherited Alzheimer Network (DIAN), ${ }^{1}$ Washington University School of Medicine, St. Louis, MO, USA; ${ }^{2}$ University of Michigan, Ann Arbor, MI, USA.

Contact e-mail: mathurinn@npg.wustl.edu

Background: Changes in Alzheimer's disease (AD) biomarkers can be detected before cognitive decline using neuroimaging and CSF measures. In autosomal dominant AD (ADAD), PET imaging biomarkers of amyloidosis and hypometabolism (measured with Pittsburgh compound $\mathrm{B}$ [PiB] and fluorodeoxyglucose [FDG], respectively) are detectable 15 and 10 years before any symptoms develop. A new marker of neurodegeneration, increased CSF visinin-like protein 1 (VILIP-1), may predict cognitive decline and has been shown to be increased in carriers of an ADAD mutation. We wanted to further characterize in ADAD this new marker of neurodegeneration by evaluating its relationship with well-known AD biomarkers, in particular FDG hypometabolism, which is believed to reflect neuronal dysfunction and injury. Methods: Participants from the Dominantly Inherited Alzheimer Network (DIAN) study $(n=202$, including 124 mutation carriers [MC], 43 of whom were symptomatic) underwent MRI, PiB and FDG PET scans and CSF VILIP-1 measurement. Dementia severity was defined by the Clinical Dementia Rating (CDR). The cohort was divided by mutation status (non-carriers [NC] vs. MC) and $\beta$-amyloid status (PiB-negative vs. PiB-positive) for group comparisons. The standardized uptake value ratio (SUVR) of PiB and FDG, obtained from an MRIbased PET processing method, were corrected and normalized to the brainstem. Partial correlations between PET SUVR data and CSF-VILIP-1 levels controlling for age and family history were performed. Results: This study confirmed increased CSF VILIP-1 levels and decreased FDG SUVR values in MC compared to NC. Among carriers, PiB-positive participants had higher VILIP-1 levels compared to PiB-negative $(\mathrm{p}<0.005)$. VILIP-1 trended higher in PiB-positive asymptomatic participants compared to PiB-negative $(\mathrm{p}=0.07)$. A positive correlation between mean cortical PiB SUVR and VILIP-1 $(r=0.36, p<0.0001)$ and a negative correlation between FDG PET in the precuneus and VILIP-1 in all participants $(r=-0.33, p<0.0001)$ were observed. The negative correlation between CSF-VILIP-1 and FDG PET was driven by the PiB-positve MC participants. Conclusions: These preliminary results show that increased CSF VILIP-1, a marker of neuronal
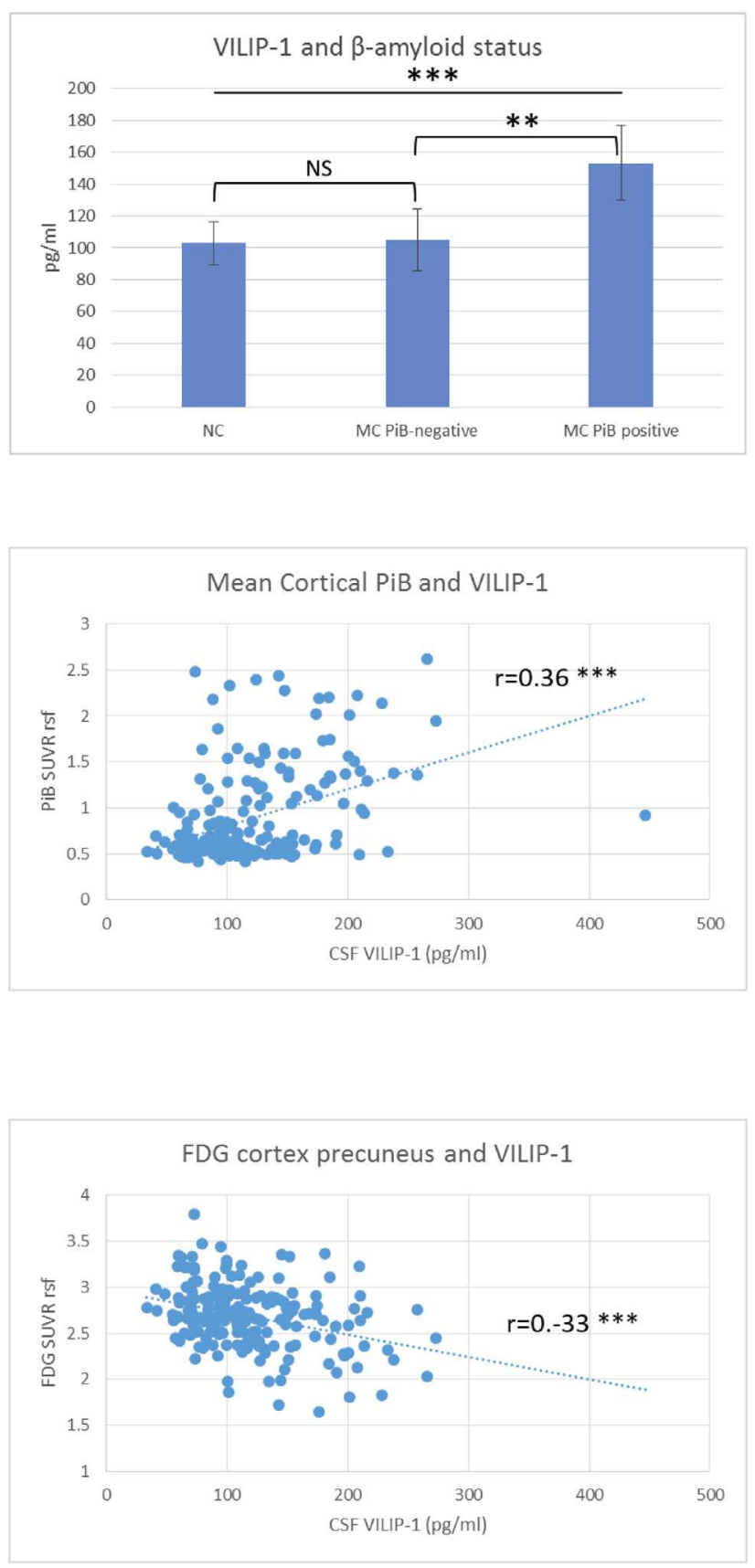

injury, is associated with both abnormal amyloid deposition and cerebral glucose hypometabolism in the DIAN population. Longitudinal investigation and evaluation of other AD features are still needed. Support: NIH/NIA U01AG032438.

\section{O2-08-06 CSF ANALYSIS DETECTS CEREBRAL B-AMYLOID ACCUMULATION EARLIER THAN AMYLOID PET}

Sebastian Palmqvist ${ }^{1}$, Niklas Mattsson ${ }^{2}$, Oskar Hansson ${ }^{2}$, Alzheimer's Disease Neuroimaging Initiative, ${ }^{1}$ Lund University, Malmö, Sweden; ${ }^{2}$ Clinical Memory Research Unit, Lund University, Lund, Sweden. Contacte-mail: sebastian.palmqvist@med.lu.se

Background:Cerebral accumulation of $\beta$-amyloid $(A \beta)$ is thought to be the starting mechanism in Alzheimer's disease (AD). 